followed by plasma exchange. Then given high dose oral corticosteroids with slow tapering and intravenous Cyclophosphamide four weekly. Significant neurological improvement noted over next 8 weeks with patient being alert and participating in ongoing multidisciplinary rehabilitation for hemiplegia.

Conclusion A case of cerebral biopsy confirmed, CSF and serum antibody-negative encephalitis is presented.

\section{SENSORY NERVE ABNORMALITIES IN MOTOR NEURON DISEASE}

Allycia MacDonald*, Merrilee Needham, Anthony Alvaro. Department of Neurology, Fiona Stanley Hospital, Murdoch, WA, Australia

\subsection{6/jnnp-2019-anzan.66}

Introduction Electrodiagnostic evaluation is crucial in establishing the diagnosis of motor neuron disease (MND) and excluding other pathologies. It is recommended that sensory nerve conduction studies (NCS) include the ulnar and sural nerves, and generally accepted that sensory nerves are normal in MND. There are however previous reports in the literature documenting variable sensory abnormalities in patients with MND. We sought to determine the frequency of unexplained sensory abnormalities seen on NCS in patients with MND.

Methods Medical records of patients attending our tertiary MND clinic over a 2 year period were reviewed. We identified 92 patients with a clinical diagnosis of MND for whom electrodiagnostic studies were available to review. Sensory abnormalities in patients without a clear underlying aetiology (eg. compressive neuropathies, diabetes) were considered unexplained.

Results Unexplained sensory abnormalities were detected in at least one nerve in 18/92 (20\%) patients. In 17 of those 18 patients, the ulnar sensory response was abnormal. 12 of 18 patients demonstrated abnormalities in 2 or more sensory nerves. Sensory abnormalities were present in 4 of 37 (10.8\%) patients with bulbar onset MND and 14 of 55 (25.4\%) patients with limb onset MND. Sensory symptoms were infrequently reported and did not correlate with abnormalities found on NCS.

Conclusions Unexplained sensory nerve action potential abnormalities are not uncommon in MND, with ulnar sensory responses the most frequently affected. These findings raise the possibility of sensory nerve pathology in patients with MND and suggest that the presence of unexplained sensory abnormalities should not exclude a diagnosis of MND.

\section{GLIAL FIBRILLARY ACIDIC PROTEIN (GFAP) ASTROCYTOPATHY ASSOCIATED WITH CEREBRAL MICRO-INFARCTION AND POOR THERAPEUTIC RESPONSE}

${ }^{1}$ Allycia MacDonald*, ${ }^{1}$ James Triplett, ${ }^{1}$ Srimathy Vijayan, ${ }^{2}$ Michael Bynevelt, ${ }^{3}$ Rahul Lakshmanan, ${ }^{1}$ Thomas Chemmanam. 'Department of Neurology, Sir Charles Gairdner Hospital, Nedlands, WA, Australia; ${ }^{2}$ Neurological Intervention and Imaging Service of Western Australia, Sir Charles Gairdner Hospital, Nedlands, WA, Australia; ${ }^{3}$ Department of Neuroradiology, Perth Children's Hospital, Nedlands, WA, Australia

10.1136/jnnp-2019-anzan.67
Introduction Glial fibrillary acidic protein (GFAP) astrocytopathy is a lesser recognised immune-mediated meningo-encephalomyelitis, which is steroid responsive in the majority of cases. Neuroimaging is unique with a distinctive symmetric white matter perivascular linear and punctate enhancement pattern. We present a case with classical phenotype but delayed clinical response, and highlight the importance of early recognition and treatment.

Case A 59-year-old Caucasian female presented with a two month history of headache, gait disturbance, insomnia, agitation, disorientation and reduced oral intake. Examination revealed a high frequency upper limb tremor, hypertonicity and pathologically brisk reflexes with impaired cognitive function. MRI brain and spinal cord demonstrated high T2 signal and striking perivascular and punctate enhancement in supratentorial white matter, cervical and upper thoracic cord. CSF examination revealed lymphocytic pleocytosis and elevated protein. Brain biopsy demonstrated reduced GFAP expression, perivascular T-lymphocytic infiltrate, and recent white matter microinfarction. CSF and serum GFAP antibodies were positive.

Motor deterioration accompanied progression to a stuporous state. High dose corticosteroids were commenced, followed by intravenous immunoglobulin and mycophenolate. While there was marked improvement of perivascular contrast enhancement on imaging, the patient continued to demonstrate prominent tremor, gait disturbance and behavioural issues 9 months following symptom onset.

Conclusions The persistence of disability in this case is likely the result of axonal loss from the initial insult, reflected by the biopsy evidence of microinfarction. Awareness of the unique pattern on MRI and the clinical phenotype will aid in early recognition and prompt treatment of this condition, thus preventing the potential long term morbidity.

\section{THE INCIDENCE, DIAGNOSIS AND OUTCOMES OF IDIOPATHIC INTRACRANIAL HYPERTENSION IN THE SOUTHERN TASMANIAN CATCHMENT}

Natasha Krishnadas*, Bruce Taylor. Neurology, Royal Hobart Hospital, Hobart, TAS, Australia

\subsection{6/jnnp-2019-anzan.68}

Introduction This study aimed to identify the incidence of idiopathic intracranial hypertension (IIH) in Southern Tasmania, Australia. Secondary aims were to elucidate demographics, current approaches to investigation, treatment and outcomes. To our knowledge, similar regional studies have not been performed.

Methods The study was approved by the University of Tasmania Human Ethics Committee. Patients presented between June 2016-June 2018 to Royal Hobart Hospital, the single tertiary Neurology service in Tasmania. Cases were identified by screening lumbar punctures (LP) performed by Neurology services (inpatient, outpatient, Radiologically-assisted) and by surveying all regional Neurologists. Medical records were used to corroborate LP results and determine whether patients met Modified Dandy Criteria (MDC) (used to define IIH in current literature). Regional population statistics were obtained from the Australian Bureau of Statistics (ABS). Duplicate records were excluded. Exclusion criteria included age $<18$ at 
data collection, LP opening pressure $<20 \mathrm{~cm}$ of water or secondary causes for IIH.

Results 45 cases identified; 30 within the Greater Hobart region, population of 229,088 (June 2016-June 2017). Cumulative incidence was $6.55 / 100,000$ (incidence rate $0.06 / 1000$ ) with classification based on Neurologist diagnosis and 5.46/ $100,000(0.05 / 1000)$ with classification according to MDC. $100 \%$ of the cohort were female. Mean age was 26.7 (range $17-45$ ) and mean weight was $105.3 \mathrm{~kg}$ (range 78-170). Headache was the most commonly reported symptom. $8.9 \%(4 / 45)$ of the total cohort were medically refractory (requiring VP/LP shunting). Ophthalmology services initiated 51\% (23/45) of the referrals.

Conclusions Our incidence rates are higher than rates in previous studies for population subsets of young women.

\section{LYMPHOMA: A GREAT IMITATOR IN NEUROLOGY AND ITS MANY FACES}

${ }^{1}$ Wei Z Yeh*, 'Subramanian Muthusamy, ${ }^{2}$ Penny McKelvie, ${ }^{1}$ Steven Collins, ${ }^{1}$ Ann French, ${ }^{3}$ Robin Filshie, 'Katrina Reardon. 'Department of Neurology, St Vincent's Hospital Melbourne, Fitzroy, VIC, Australia; 'Department of Pathology, St Vincent's Hospital Melbourne, Fitzroy, VIC, Australia; ${ }^{3}$ Department of Haematology, St Vincent's Hospital Melbourne, Fitzroy, VIC, Australia

\subsection{6/jnnp-2019-anzan.69}

Introduction The label 'great imitator' refers to conditions which can cause varied manifestations and mimic diseases. Lymphoma is worthy of this title. We present three cases.

Cases 1: 66-year-old-man with progressive vertical diplopia and unsteady gait over four weeks. MRI brain and spine demonstrated a supratentorial para-falcine soft tissue lesion, mid-thoracic cord enhancement and right axillary mass. Serum ACE was elevated. Serum HIV serology was positive. Right axillary mass core biopsy diagnosed Burkitt lymphoma.

2: 50-year-old man with a 4-week history of constitutional symptoms on a background of ITP and splenomegaly. During admission he developed urinary retention, bilateral lower limb weakness and numbness and confusion. Infectious and vasculitic screens were unremarkable. CT chest, abdomen and pelvis demonstrated splenomegaly. CSF and bone marrow analyses were non-diagnostic. A random skin biopsy diagnosed intravascular lymphoma.

3: 65-year-old man with two weeks of headache and diplopia on a background of previously treated Burkitt lymphoma. CSF analysis showed $45 \times 10^{6} / \mathrm{L}$ white cells and glucose $<0.6 \mathrm{mmol} / \mathrm{L}$. Cytologic analysis was negative for malignancy. Bacterial culture and Cryptococcal antigen were negative. FDG-PET dramatically showed disseminated spinal and cranial leptomeningeal disease. MRI brain showed thin dural thickening correlating to area of increased uptake on PET. He was diagnosed with Burkitt lymphoma relapse and treated with chemotherapy and autologous stem cell transplant.

Conclusion The varied manifestations in our cases demonstrate the ability for lymphoma to mimic infective, inflammatory, granulomatous (including sarcoidosis) and neoplastic aetiologies. When the diagnosis is uncertain, the possibility of this great imitator should be considered.

\section{THE GASTROINTESTINAL MICROBIOME IN PARKINSON'S DISEASE: IMPACTS OF MOTOR AND NON-MOTOR FEATURES, MEDICATIONS, LIFESTYLE AND DIET}

${ }^{1}$ Michal Lubomski*, ${ }^{2}$ Ai Huey Tan, ${ }^{2}$ Shen-Yang Lim, ${ }^{3}$ Andrew Holmes, ${ }^{1}$ Ryan L Davis, ${ }^{1}$ Carolyn M Sue. ${ }^{1}$ Department of Neurogenetics, Kolling Institute, University of Sydney and Royal North Shore Hospital, Sydney, NSW, Australia; ${ }^{2}$ Neurology Department, Mah Pooi Soo and Tan Chin Nam Centre for Parkinson's and Related Disorders, Faculty of Medicine, University of Malaya, Kuala Lumpur, Malaysia; ${ }^{3}$ School of Life and Environmental Sciences, The Charles Perkins Centre, University of Sydney, Sydney, NSW, Australia

\subsection{6/jnnp-2019-anzan.70}

Introduction The human gastrointestinal microbiome (GM) has been proposed to be integral in the pathogenesis of Parkinson's disease (PD). Evidence supports a bidirectional interaction between the brain and the gut that is mediated by the GM. Dysbiosis of the GM is believed to negatively influence vital physiological functions in many diseases.

Methods We reviewed the literature on changes in human physiological function associated with gut microbial community states in PD. In particular, we evaluated the literature for effects of GM dysbiosis on motor and non-motor features, dietary and lifestyle factors and medication use in PD.

Results Altered GM profiles in PD have been suggested to disrupt vital signalling pathways within the microbiota-gutbrain axis, integral to regulating physiological digestive function and metabolic homeostasis. Unfavourable variations in the GM have been shown to perturb mood (anxiety/ depression), cognition, perception (hallucinations/delusions), gastrointestinal motility, including constipation in PD. Further, varied clinical motor phenotypes, including postural instability and gait disturbance have been attributed to alterations in the GM, in addition to the use of catechol-Omethyltransferase inhibitors, anticholinergics and levodopa. Variations in dietary and lifestyle factors have also been inferred to cause alterations in GM profiles, including caffeine consumption, macronutrient intake, smoking and the effects of ageing and exercise.

Conclusions It is apparent from the mounting evidence that alterations in the GM are intimately involved in PD pathogenesis. However, the GM can also be modulated by dietary, lifestyle and treatment factors that may influence motor and nonmotor features as well as disease progression.

\section{NOVEL WIRELESS S - PATCH DEVICE MAY EFFICIENTLY DETECT ATRIAL FIBRILLATION IN PATIENTS WITH ISCHAEMIC STROKE}

${ }^{1}$ Suzana Lazarovska* ${ }^{1} \mathrm{Hui}$ Tie, ${ }^{1}$ Andrew Hopkins, ${ }^{1}$ Hany Dimitri, ${ }^{2}$ Upul Premawardhana, ${ }^{3}$ Alan Mcdougall, ${ }^{4}$ Sumana Gopinath, ${ }^{5}$ Josephine Chow, ${ }^{1}$ Rohan Rajaratnam. ${ }^{1}$ Cardiology, Liverpool Hospital, Sydney, NSW, Australia; ${ }^{2}$ Cardiology, Campbelltown Hospital, Sydney, NSW, Australia; ${ }^{3}$ Neurology, Liverpool Hospital, Sydney, NSW, Australia; ${ }^{4}$ Neurology, Campbelltown Hospital, Sydney, NSW, Australia; ${ }^{5}$ Liverpool Renal Clinical Research Centre, Co-Director, Sydney, NSW, Australia

\subsection{6/jnnp-2019-anzan.71}

Introduction Atrial fibrillation (AF) is the most common atrial arrhythmia leading to increased risk of thromboembolic events. Ward telemetry (WT) has a low detection rate for AF in stroke patients and often patients are not appropriately treated with anticoagulation. This study compares conventional 2-day WT versus 4 -day wireless $S$-patch monitoring to detect AF. 